\title{
Explorando el sistema de protección de la niñez en Honduras
}

\author{
Martha Lorena Suazo, Ph.D. \\ Universidad Nacional Autónoma de Honduras, Honduras* \\ Kevin Alberto Cruz, Lic. \\ Universidad Nacional Autónoma de Honduras, Honduras ${ }^{\star \star}$ \\ Henry Parada, Ph.D. \\ Universidad de Ryerson, Honduras ${ }^{\star \star}$
}

suazomlorena@yahoo.com

\section{Resumen (analítico)}

Se exploran las prácticas institucionales que facilitan u obstaculizan la protección de derechos de niños, niñas y adolescentes en el sistema de protección de la niñez en Honduras a través de sus diferentes etapas. Desde un diseño de Etnografía Institucional, se realizaron entrevistas a catorce trabajadores de instituciones que trabajan con niñez, y a tres residentes en un domicilio de protección. Como resultado, se identificaron múltiples instancias de denuncia, y una estrecha comunicación entre las mismas. Sin embargo, existe carencia de recursos en el sistema, y prejuicios contra adolescentes de la comunidad LGTBIQ; así como prácticas alimentadas por una concepción tradicional de la niñez. El sistema no solamente parece incumplir con algunos principios de la Convención, sino que se encuentra desbordado por precarias condiciones del contexto nacional.

\section{Palabras clave}

Código de la Niñez y Adolescencia, Convención de los Derechos del Niño; etnografía institucional; Honduras; infancia; sistema de protección de la niñez.

\section{Thesauro}

Tesauro de Ciencias Sociales de la Unesco.

\section{Para citar este artículo}

Suazo, M. L., Cruz, K. A., \& Parada, H. (2020). Explorando el sistema de protección de la niñez en Honduras. Revista Latinoamericana de Ciencias Sociales, Niñez y Juventud, 18(2), 1-26. http://dx.doi.org/10.11600/1692715x.18207

\section{Historial}

Recibido: 21.10 .2019

Aceptado: 10.02 .2020

Publicado: 26.05.2020

Información artículo

El artículo forma parte de los resultados del estudio «Prácticas Institucionales en el sistema de protección de la niñez en Honduras», el cual constituye uno de los ejes de investigación del macroproyecto «Asociación por los Derechos de la Niñez y la Juventud» realizado por el Instituto de Investigaciones Sociales (IIS) de la Universidad Nacional Autónoma de Honduras con apoyo de la Escuela de Trabajo Social de la Universidad de Ryerson en Canadá. El financiamiento es realizado por el Consejo de Investigación en Ciencias Sociales y Humanidades de Canadá según archivo No. 895-2015-1014. Los datos presentados se recolectaron entre marzo y noviembre de 2018. Área: Ciencias Sociales. Sociología. Subárea: Temas Sociales. 


\section{Exploring the child protection system in Honduras}

Abstract (analytical)

This study explores institutional practices that either facilitate or hinder the protection of the rights of children and adolescents in the child protection system in Honduras. The research focuses on the different stages that users go through when they request services. Using an institutional ethnography design, semi-structured interviews were held with 14 workers from institutions that work with users in the system, as well as with three adolescents living in a protection home were conducted. The main results found that there are multiple mechanisms for reporting cases of child abuse, as well as close communication between the different mechanisms. However, there is a lack of resources in the system as well as prejudice against adolescents from the LGTBIQ community; also, practices are fed by a traditional conception of childhood. The system doesn't just violate some of the principles of the Convention on the Rights of the Child, but that its work is overwhelmed by the precarious conditions in the national context.

Keywords

Code of childhood and adolescence, Convention on the Rights of the Child, child protection system, childhood, Honduras, Institutional ethnography.

\section{Explorando o sistema de proteção infantil em Honduras}

Resumo (analítico)

Este estudo teve como objetivo explorar práticas institucionais que facilitam ou dificultam a proteção dos direitos das crianças e adolescentes no sistema de proteção à criança em Honduras, através das diferentes etapas. Para isso, com base no desenho de Etnografia Institucional, foram realizadas entrevistas semiestruturadas com quatorze trabalhadores de instituições governamentais e não governamentais e com três adolescentes residentes em um lar de proteção. Entre os principais resultados, constatou-se que existem várias instâncias para relatar casos de abuso infantil, bem como uma estreita comunicação entre elas. No entanto, havia também falta de recursos nas instituições que compõem o sistema, bem preconceitos contra adolescentes da comunidade LGTBIQ. Na análise, muitas práticas são alimentadas por uma concepção tradicional da infância. Por outro lado, o sistema de proteção à criança parece não apenas violar alguns dos princípios da Convenção, mas que seu trabalho é sobrecarregado por precárias condições do contexto nacional.

\section{Palavras-chave}

Código da Infância e Adolescência, Convenção sobre os Direitos da Criança, etnografia institucional, Honduras, infância, sistema de proteção à criança.

Información autores

[*] Doctora en Ciencias Sociales con orientación en Gestión del Desarrollo por la Universidad Nacional Autónoma de Honduras (Unah). Profesora en el Departamento de Trabajo Social de la Unah. Correo electrónico: suazomlorena@yahoo.com; martha.suazo@unah.edu.hn (iD) 0000-0003-3083-0374.

[**] Candidato a Máster en Sociología por la Unah. Asistente de Investigación en el Instituto de Investigaciones Sociales y Docente en el Departamento de Sociología de la Unah. Miembro del Grupo de Trabajo en Infancias y Juventudes de Clacso. iD 0000-0001-8327-0150. Correo electrónico: cruzkevin@unah.hn; cruz.kevin@unah.edu.hn

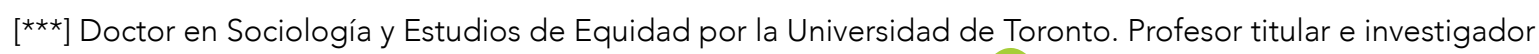
en la Escuela de Trabajo Social de la Universidad de Ryerson, Canadá. (iD 0000-0003-0213-3117. Índice H5: 8. Correo electrónico: hparada@ryerson.ca 


\section{Introducción}

esde la década de los noventa, los gobiernos de América Latina han tratado de
incorporar los principios de la Convención de los Derechos del Niño y la Niña en sus respectivas legislaciones y sistemas de protección de la niñez. Sin embargo, aunque este hito es reconocido tanto por la comunidad académica como por profesionales que trabajan con la infancia como un importante avance hacia un cambio de paradigma para entender a los niños, niñas y adolescentes como sujetos de derechos en vez de objetos pasivos de intervención, en la práctica no significa que las representaciones sobre la infancia y sus derechos hayan cambiado (Alzate-Piedrahíta, 2003). Al contrario, diversos reportes y estudios en la región (Martínez-Franzoni, 2014; Parada, 2011; Sola-Morales \& Campos-Garrido, 2019; Villalta \& Llobet, 2015; Visión Mundial, 2014) señalan la brecha existente entre lo que dicen las políticas y leyes nacionales relacionadas con niñez — las que, a su vez, se fundamentan en la Convención-y lo que verdaderamente ocurre en la práctica de las instituciones responsables de ofrecer protección a la niñez y adolescencia, donde todavía prevalecen concepciones tradicionales sobre este grupo poblacional.

Para autoras como Di Loro (2010) «la derogación, desde el punto de vista declarativo, de viejos paradigmas sobre el modo de intervenir en la infancia, no implica (...) la transformación de las prácticas, en tanto que estas se producen sobre el trasfondo de creencias sociales compartidas» (p. 148). Así mismo, el «conocimiento» predominante que informa el trabajo realizado por profesionales de la psicología, el trabajo social o el derecho dentro del sistema se deriva exclusivamente de perspectivas adultas (Mayall, 2001). De esta manera, se puede afirmar que lo dispuesto en las legislaciones nacionales y los reglamentos que delimitan el trabajo de las instituciones que conforman los sistemas de protección de la niñez no se ve necesariamente reflejado en las prácticas institucionales, ya que este no es el único referente utilizado por los trabajadores para orientar su quehacer. Más aún, siguiendo a Villalta y Llobet (2015), es en las redes de relaciones entre actores dentro de los sistemas de protección donde debe buscarse el carácter específico de la protección de derechos. Partiendo de estas premisas, este estudio trata de respon- 
der a la pregunta, ¿cuáles son las prácticas institucionales, normadas por la legislación nacional, así como las desarrolladas en la cultura de cada institución, que facilitan u obstaculizan la protección de los derechos de las niñas, niños y adolescentes en el sistema de protección de la niñez en Honduras?

El trabajo se fundamenta en la nueva sociología de la infancia, campo que - sobre todo desde los años 200o- ha adquirido una progresiva legitimidad en el mundo de la sociología hispano-hablante (Voltarelli et al., 2018). Siguiendo este paradigma, se parte del entendimiento de la niñez como una construcción social (Prout \& James, 1997), pero también comprendiéndola desde una mirada relacional. Es decir, tomando como referencia dos de los tres enfoques clásicos ${ }^{1}$ en este campo de la sociología (Gaitán-Muñoz, 2006). En este sentido, se considera que los niños, niñas y adolescentes han sido asignados a un orden social que, en términos generacionales, los ubica como un grupo subordinado a la población adulta, permitiendo que sus vidas se encuentren significativamente condicionadas por la voluntad de los segundos (Mayall, 2001). De esta manera, incluso los procesos de intervención en los sistemas de protección se fundamentan en visiones adultocéntricas (Mayall, 2001), especialmente aquellas que subrayan la visión de la niñez como receptáculos pasivos dentro de los que el mundo adulto, maduro, racional y pleno, deposita el conocimiento y las capacidades necesarias para convertir estos entes pre-humanos, salvajes, inmaduros e incompletos en adultos integrados en la sociedad (Prout \& James, 1997); argumento sobre el que se han fundamentado los procesos de intervención e institucionalización de la niñez históricamente (Alzate-Piedrahíta, 2003; Mayall, 2001).

Dada la importancia de la Convención para entender los procesos de construcción de la niñez en los años recientes (Alzate-Piedrahíta, 2003), el análisis de este trabajo se complementa con un enfoque crítico de los derechos humanos, específicamente desde los principios teóricos de la Escuela de Frankfurt. Para esta, los derechos humanos, a la vez que podrían considerarse como un mecanismo para renegociar poder por parte de grupos vulnerables, también pueden ser un recurso ideológico que representa a los ciudadanos como iguales mientras se fundamentan en una sociedad esencialmente desigual (Rensmann, 2017).

Haciendo una revisión del conocimiento que se ha producido en relación con los sistemas de protección de la niñez en América Latina en general, y en la región centroamericana en particular, se identifica que tanto las ciencias sociales como los organismos

\footnotetext{
${ }^{1}$ Siendo el tercero el enfoque estructural.
} 
gubernamentales han contribuido a su comprensión mediante reportes y trabajos de investigación que responden a distintas preocupaciones empíricas y académicas. Se identifican cuatro temas principales:

1. Brecha entre lo dispuesto en la Convención, las políticas públicas y la legislación nacional y su implementación en los procesos cotidianos de las instituciones que conforman los sistemas de protección de la niñez.

2. Prevalencia de una concepción tradicional de la niñez.

3. Limitación de recursos en los sistemas de protección de la niñez para llevar a cabo sus mandatos.

4. Ausencia de protocolos y personal no especializado en las instituciones del sistema.

Uno de los temas más sobresalientes es la significativa brecha entre los compromisos asumidos por los Estados a través de la Convención y las prácticas efectivas dentro de los sistemas de protección (Martínez-Franzoni, 2014; Olivares \& Reyes, 2016; Parada, 2011; Parada et al., 2007; Sola-Morales \& Campos-Garrido, 2019; Villalta \& Llobet, 2015; Visión Mundial, 2014). Como ejemplo, en un análisis de los sistemas de protección de la niñez en América Latina, Visión Mundial (2014) señala que los marcos legales que los rigen no coinciden con las realidades de los países del continente, pues «muchas de esas leyes no son aplicadas a nivel local ni responden a los contextos locales específicos» (p. 6).

Por otra parte, se identifica en el sistema la prevalencia de estigmas y concepciones tradicionales de la niñez; es decir, estos son vistos como objetos de intervención, como «menores» o como problema por parte de tomadores de decisión o profesionales de la niñez (Martínez-Franzoni, 2014; Oficina Internacional de los Derechos del Niño, s. f.; Olivares \& Reyes, 2016; Parada, 2011). Al respecto, Martínez-Franzoni (2014) desafía a

plantear políticas sociales donde se produzca una transición desde la concepción de los niños y las niñas como «menores», «partes o fragmentos» de una humanidad inconclusa a un todo sistémico, a ciudadanos, sujetos de derecho, lo que pone como tema de debate la ciudadanía infantil. (p. 12)

Así mismo, las instituciones responsables de los sistemas de protección de la niñez no cuentan con los recursos económicos para dar atención a todos los niños, niñas y familiares que lo necesitan (Martínez-Franzoni, 2014; Parada et al., 2007; Visión Mundial, 2014). Aún más, en países como Honduras, la mayor parte de la poca inversión pública 
que se destina a la niñez tiende a utilizarse en pago de salarios (Oficina Internacional de los Derechos del Niño, s. f.; República de Honduras, 2014), comprometiendo más el alcance efectivo que las instituciones y programas destinados a la niñez puedan tener.

La literatura señala también que las instituciones no cuentan con protocolos o reglamentos para definir los roles y procedimientos que los actores del sistema deben seguir para atender las necesidades de la niñez en situaciones particulares, tales como el abordaje de casos de «alto riesgo» (Parada et al., 2007), la definición de planes de intervención y seguimiento (Oficina Internacional de los Derechos del Niño, s. f.) o la estandarización de los procesos de denuncia (Santos, 2017). Por otro lado, es frecuente encontrar el señalamiento de que una cantidad significativa del personal que trabaja en dichas instituciones cuenta con poca o nula preparación académica (Martínez-Franzoni, 2014; Mcreery-Bunker et al., 2009; Oficina Internacional de los Derechos del Niño, s. f.; Parada et al., 2007).

Resulta relevante señalar que gran parte de los estudios utilizan a la Convención y su enfoque de la niñez como marco teórico para analizar los sistemas de protección (Dávila \& Naya, 2012; Martínez-Franzoni, 2014; Mieles \& Acosta, 2012; Oficina Internacional de los Derechos del Niño, s. f.; Unicef, 2016). Así mismo, una de las consistencias encontradas en la revisión de los diversos estudios es la utilización de metodologías cualitativas para recoger y analizar sus datos (Dávila \& Naya, 2012; Martínez-Franzoni, 2014; Oficina Internacional de los Derechos del Niño, s. f.; Olivares \& Reyes, 2016; Parada, 2011; Parada et al., 2007; Urzin et al., 2017; Visión Mundial, 2014). Se constató también que gran parte de los estudios o reportes realizados, particularmente en el caso de Honduras, son llevados a cabo por organismos gubernamentales y no gubernamentales; lo anterior implica muchas veces una ausencia de enfoques teóricos que permitan la acumulación del conocimiento relacionado con la niñez y sus sistemas de protección, y el abordaje de sus problemáticas desde una perspectiva afín a las discusiones actuales desde las ciencias sociales.

El presente trabajo trata de ahondar en qué consiste esa brecha identificada entre la normativa y lo que realmente ocurre en las instituciones que ofrecen protección a la niñez, haciendo énfasis en la exploración de las prácticas que facilitan u obstaculizan la protección de sus derechos en las instituciones que conforman el sistema de protección en Honduras. 


\section{Contexto social e institucional}

La niñez y adolescencia de Honduras (o a 19 años) está constituida por 3.9 millones de niños y niñas, que representan el $47 \%$ de la población nacional (Observatorio Demográfico Universitario, 2018). En consonancia con la conformación de un sistema responsable de la protección de este grupo poblacional, en 1990 el Estado de Honduras ratificó su adscripción a la Convención (Organización de las Naciones Unidas, 1989), iniciando de esta manera un proceso para enmarcar en los principios de este tratado internacional el trabajo estatal relacionado con la niñez y la adolescencia; mismo que se refleja en la creación del Código de la Niñez y la Adolescencia (República de Honduras, 1996) y del Instituto Hondureño de la Niñez y la Familia (Ihnfa) (República de Honduras, 1998), marco legislativo e instancia administrativa que encuadraron los esfuerzos de protección de la niñez en el país hasta el 2013. Entre 2013 y 2014, los Gobiernos de turno realizaron una serie de reformas al abordaje estatal de la niñez y adolescencia, cuyo resultado más visible fue la modificación de 123 artículos del Código de la Niñez y Adolescencia en 2013 (Decreto No. 35, 2013), así como la clausura del Ihnfa en 2014. Se creó en sustitución una nueva institución responsable del sistema de protección: la Dirección de Niñez, Adolescencia y Familia (Dinaf) (Santos, 2017).

Algunas de las principales consecuencias de tales reformas fueron que, en contraste con el Ihnfa, que era una institución autónoma en los procesos de decisión administrativos y presupuestarios y tenía una infraestructura de alcance nacional para ofrecer servicios de protección a la niñez, la Dinaf es una dirección subordinada a las disposiciones de la Secretaría de Desarrollo e Inclusión Social y no tiene presencia en todo el territorio nacional, puesto que solo cuenta con seis oficinas regionales para dar cobertura a los 18 departamentos del país. Además, se establece un nuevo enfoque en la atención a las necesidades de protección de la niñez, ya que se desprende a la Dinaf de la responsabilidad de atender directamente (brindando techo y satisfacción a otro tipo de necesidades básicas) a la niñez y adolescencia que por diversos tipos de vulneración requieran la tutela del Estado, trasladando esta responsabilidad a las municipalidades y organizaciones no gubernamentales. Con estas reformas se inicia un proceso de descentralización y tercerización en el sistema de protección, estipulados en mismo Plan Estratégico de la Dinaf (2015).

Encima de esto, el mandato de la Dinaf debe ser realizado en un contexto en donde más del 6o\% de la población vive en condiciones de pobreza (Banco Mundial, 2019), la cual afecta a más del 70\% de la niñez y adolescencia en el país (Saunders et al., 2014), y donde la infancia es el grupo más vulnerable desde la perspectiva de la pobreza moneta- 
ria en la región (Cepal, 2013, como es citado en Unicef, 2016). Adicionalmente, la violencia es uno de los problemas que más afectan a los niños, niñas y adolescentes ya que, Honduras, con una tasa de homicidios de 43.6 por cada 100 mil habitantes en 2017 (Instituto Universitario en Democracia, Paz y Seguridad, 2018), es un escenario altamente peligroso para ellos. Las tasas de homicidio para este grupo poblacional ascienden a zo asesinatos por cada 100 ooo habitantes (Oficina Internacional de los Derechos del Niño, s. f.).

\section{Objetivos de la investigación}

El presente estudio explora las prácticas institucionales que facilitan u obstaculizan el trabajo del sistema de protección de la niñez en Honduras. Para ello, se pretende describir: 1) las etapas que típicamente atraviesan los usuarios del sistema cuando reciben los servicios de protección; 2) las instituciones involucradas y el papel que desempeñan en las diferentes etapas; $y, 3$ ) la interacción entre las diferentes instituciones que integran este sistema.

\section{Metodología}

En consonancia con el enfoque construccionista de la sociología de la infancia (Gaitán-Muñoz, 2006) el método de investigación del presente estudio fue la etnografía institucional, que consiste en adentrarse en las prácticas de un sistema; es decir, en las acciones, procesos y discursos que tienen lugar en el marco de una institución para dar forma a las relaciones que regulan su trabajo cotidiano (Devault, 2006). Estas acciones y procesos no necesariamente son el reflejo de discursos oficiales plasmados en marcos legislativos o reglamentos, sino que en muchas ocasiones difieren de ellos (Parada, 2011). El conocimiento elaborado a partir de este método se construye desde la propia experiencia de los informantes, tal como es vivida por ellos desde la posición que ostentan en los procesos cotidianos que deben llevar a cabo para cumplir con sus roles institucionales, sean estos como trabajadores o como usuarios. La razón por la cual se adoptó este abordaje obedece a que, en los procesos de la cotidianidad institucional, los trabajadores del sistema pueden resignificar el marco legislativo de su rol institucional, actuando así en buena medida conforme a su propia interpretación de los protocolos del sistema de protección (Villalta \& Llobet, 2015). Aún más, ante la ausencia de protocolos y otros referentes normativos en el cuidado de la niñez (por ejemplo, debido a la reestructuración del sistema de protección), los trabajadores pueden recurrir a elementos idiosincráticos que 
orienten su interacción con los niños y familiares que solicitan los servicios del sistema. En este sentido, resulta de vital importancia conocer las prácticas que efectivamente se llevan a cabo en la vida cotidiana de las instituciones e identificar en qué medida se acercan o no lo estipulado por los textos y discursos legales.

Para realizar el estudio, se conformó un equipo de miembros y colaboradores del Instituto de Investigaciones Sociales de la Universidad Nacional Autónoma de Honduras, integrado por distintas disciplinas como el trabajo social, la sociología, la demografía y la psicología.

\section{Participantes}

Si bien el interés de la etnografía institucional se concentra en las prácticas institucionales, el punto de partida para acceder a ellas son los trabajadores y usuarios del sistema, aunque estos no son el objeto de estudio mismo. Los participantes que brindaron información sobre dichas prácticas fueron seleccionados siguiendo los siguientes criterios: 1) que trabajaran en instituciones estatales y no estatales del sistema de protección de la niñez; 2) que en su trabajo cotidiano tuvieran interacción cara a cara con los niños o familiares que solicitan los servicios de la institución; 3) en el caso de los adolescentes, que fueran residentes de alguno de los domicilios que se ofrecen como medida de protección dentro del sistema. Por razones éticas y legales fueron excluidos del estudio los niños y niñas menores de 12 años. Como resultado del proceso, en el período de marzo a noviembre del 2018, se entrevistó a 17 participantes (tabla 1).

\section{Tabla 1}

\section{Caracterización de participantes}

\begin{tabular}{ccccc}
\hline \multirow{2}{*}{ Sector } & Institución & Adultos & NNA & Total \\
\hline \multirow{3}{*}{ Estatal } & Dinaf & 6 & 0 & 6 \\
\cline { 2 - 5 } & Policía Nacional & 2 & 0 & 2 \\
\cline { 2 - 5 } & Ministerio Público & 2 & 0 & 2 \\
\cline { 2 - 5 } No estatal & Centro educativo & 2 & 0 & 2 \\
\cline { 2 - 5 } & ONG & 1 & 3 & 4 \\
\hline \multirow{2}{*}{ Total } & Centro de paso & 1 & 0 & 1 \\
\hline
\end{tabular}

Nota. NNA: niños, niñas y adolescentes. 
Los datos del estudio se recolectaron en dos ciudades: Distrito Central, en el departamento de Francisco Morazán, en la región central de Honduras, y San Pedro Sula, en el departamento de Cortés, en la región noroccidental del país. Cabe resaltar que el 35\% de los participantes ( $n=6$ ) trabajan en la Dinaf. Esto debe ser tomado en cuenta al interpretar los resultados, por un lado, porque su trabajo y discurso tiende a reflejar la versión oficial del sistema, y por el otro, porque su interacción directa con niños niñas y adolescentes se concentra en las primeras etapas de protección, es decir, en los procesos administrativos de denuncia y derivación a las instituciones que toman la responsabilidad en las siguientes etapas.

\section{Técnicas de recolección de datos}

Se realizaron entrevistas semiestructuradas con los proveedores de servicios y usuarios que conforman el sistema de protección de la niñez. Adicionalmente, se realizó un trabajo de observación en las instituciones visitadas. Desde el método de la etnografía institucional no se construye un instrumento en sentido estricto, sino una guía de preguntas que permiten iniciar la exploración de las prácticas institucionales desde el conocimiento de los propios actores. Esto implica conocer qué hacen los actores y hacia quiénes se dirigen sus prácticas en el proceso de ofrecer protección a niños, niñas y adolescentes.

Este estudio recibió la aprobación del Comité de Ética de la Universidad de Ryerson. En el marco de este protocolo, el equipo de investigación solicitó consentimiento informado a las y los participantes para dejar registro de las entrevistas en un archivo de audio.

\section{Proceso de análisis datos}

En un primer momento se procedió a realizar un mapeo de los procesos y prácticas identificados en las grabaciones de las entrevistas, pero también de las conexiones entre estas dentro de cada institución y de las relaciones existentes entre las diferentes instituciones que conforman el sistema de protección de la niñez. De esta manera, se logró un primer acercamiento a la comprensión de su complejo funcionamiento. El mapa fue desarrollado utilizando un software especializado en la elaboración de diagramas llamado Lucidchart (https://www.lucidchart.com).

Seguidamente, se procedió a transcribir las grabaciones de las entrevistas, las cuales fueron codificadas en tres etapas superpuestas con el apoyo del software de análisis cualitativo $\mathrm{NVivo}^{\mathrm{TM}}$ (v. 12). En la primera, llamada codificación abierta (Strauss \& Corbin, 2002), se organizó toda la información relevante en códigos que representaran la diversidad de 
afirmaciones, acciones, procesos, prácticas y dimensiones más relevantes. En la segunda etapa, llamada codificación axial, se procedió a organizar los códigos identificados en torno a categorías de mayor alcance, capaces de aglutinar procesos más generales. Finalmente, la codificación temática, permitió integrar los códigos axiales en categorías de mayor abstracción y, de esta manera, elevar el nivel comprensivo del sistema de protección.

\section{Resultados}

A partir de los datos recolectados se elaboró un mapa que ilustra los diferentes caminos que sortean los usuarios del sistema de protección para utilizar los servicios que este provee, así como las prácticas institucionales que concretizan los objetivos en cada una de las etapas. La información recolectada se clasificó en torno a cuatro temas principales: 1) ingreso al sistema; 2) medidas implementadas; 3) seguimiento de medidas; $y$, 4) salida del sistema. Cabe mencionar que el ingreso al sistema por parte de los niños, niñas o adolescentes es resultado de la denuncia de cualquier tipo de maltrato en su contra ante alguna institución competente, sea esta gubernamental o no gubernamental.

\section{Ingreso al sistema}

Como puede apreciarse en la figura 1, existen múltiples puntos de entrada para los usuarios del sistema de protección de la niñez. Se identificó que los centros educativos, la Policía Nacional, el Ministerio Público y la propia Dinaf son instituciones centrales que sirven como punto de ingreso.

Durante esta etapa, el trabajo de los proveedores de servicios pasa por identificar si ha ocurrido maltrato contra un niño, niña o adolescente o, como se denomina en el lenguaje institucional, una vulneración en sus derechos.

Hasta este momento, cuando alguien llega con una denuncia, se atiende en el área de filtro; en el área de filtro se le escucha al usuario (...). Porque el área de filtro es la que dice «bueno, si, debemos atender este caso» o «no, le vamos a orientar y conducir hacia el área correcta, mediante el proceso judicial» (...). Ese es todo el proceso; obviamente no está escrito en piedra que va a ser así, como de forma lineal cada caso. (Directivo Dinaf, septiembre, 2018) 
Figura 1

Puntos de ingreso al sistema de protección de la niñez en Honduras

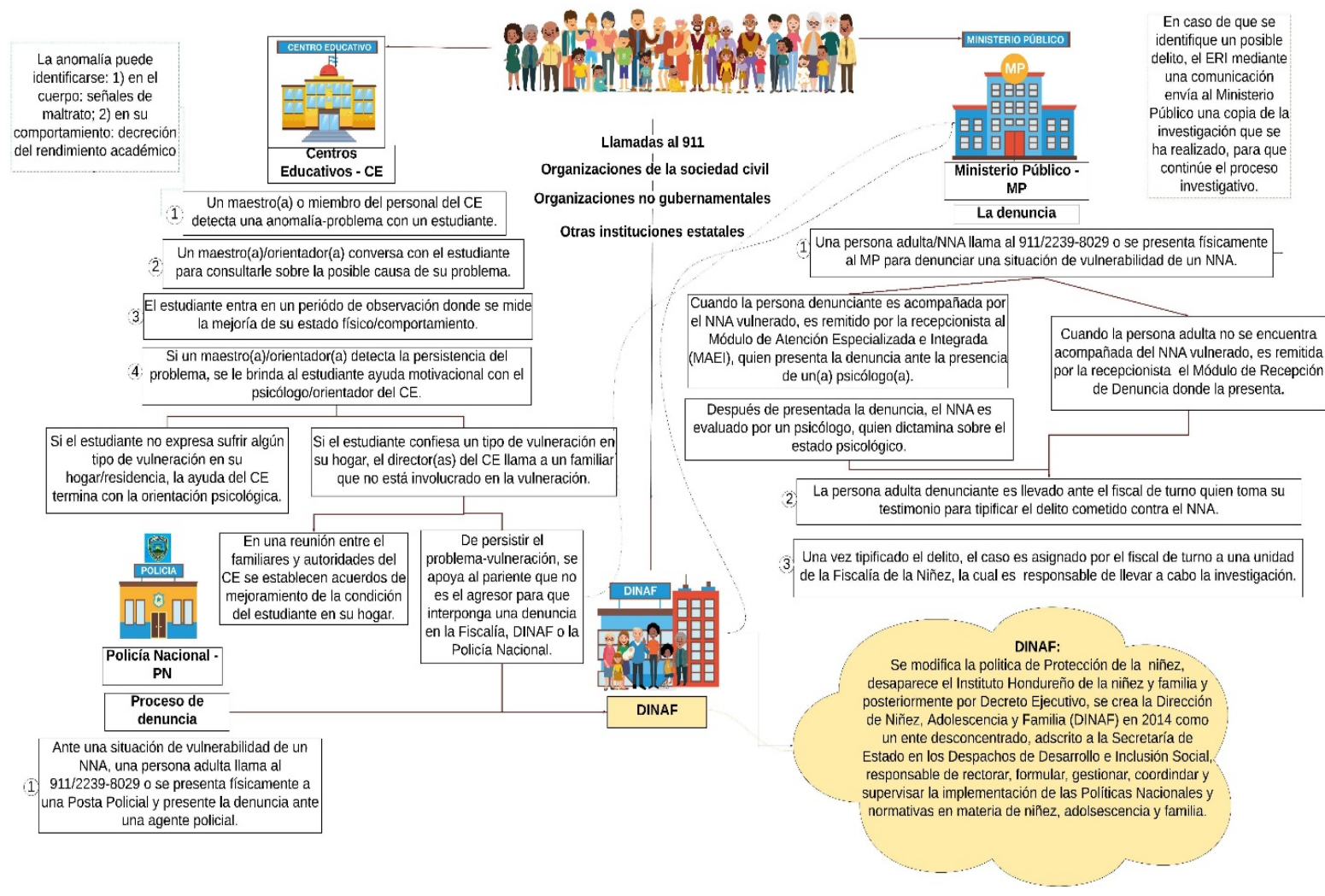

En el caso de los centros educativos, tal como puede apreciarse en el mapa (figura 1), se presentan una serie de circunstancias que tienen como principal actor al maestro o al consejero escolar, quien identifica anomalías o problemas con un estudiante por medio de señales de maltrato en su cuerpo o cambios drásticos en su comportamiento. Como respuesta, lleva a cabo una charla personal en la cual se da un espacio al niño o niña para que pueda manifestar si existe algún problema en su hogar. Si el maestro o consejero no recibe una confirmación por parte del estudiante, el proceso finaliza. Por otra parte, si el maestro/consejero logra identificar algún tipo de problema en el hogar del niño o niña, en una reunión con las autoridades del centro educativo y los familiares del joven se acuerdan medidas para solventar la situación. Sin embargo, luego de un período de prueba, si la autoridad del centro detecta la persistencia de la problemática o identifica la posible ocurrencia de un delito, apoyan al niño, niña o adolescente y a un familiar que no sea el causante del maltrato para que interpongan una denuncia en la Policía, en la Fiscalía de la Niñez o en la Dinaf. 
En relación con el Ministerio Público, los niños, niñas o adolescentes pueden ingresar al sistema de protección si alguien reporta que se encuentran de manera irregular fuera del país, ante lo cual esta institución inicia un proceso de retorno. También pueden ingresar mediante denuncia de maltrato o a causa del proceso de investigación de un delito.

Cuando los niños son llevados a otros países, hay una sección aquí especializada, que se llama «Sección de alertas amarillas». La abogada [nombre] es la encargada de este tema, y es justamente cuando padres se llevan a sus hijos sin el permiso de su otro padre; de repente por puntos ciegos o con identificaciones falsificadas, y cuando ya se detecta que el niño o niña está en otro país, entonces empieza a funcionar la investigación a través de una alerta amarilla para ubicar al niño y la niña, y tratar de repatriarlo. (Fiscal, marzo, 2018)

Por parte de la Policía Nacional el ingreso al sistema es frecuente a causa de la captura de un niño, niña o adolescente en condiciones de abandono, mendicidad o explotación laboral durante operativos policiales, mediante presentación de denuncias de maltrato contra ellos o por denuncias de «rebeldía» interpuestas por sus propios familiares. «Aparte de eso se hacen operativos frecuentemente, en centros nocturnos, en esos lugares que les llaman night clubs, hoteles, a veces detectan que hay niños o niñas que están siendo víctimas de ese tipo de delitos» (Fiscal, marzo, 2018).

Se pueden hacer por dos rutas, una infraganti, que llame al 911 y diga en este momento están agrediendo un menor y los vecinos dicen que no es la primera vez que se da el maltrato, entonces inmediatamente, pueden proceder y sacar al menor, esa es una ruta. Otra manera, por orden judicial, requerimiento fiscal, una orden que dice «vaya saque a ese menor», que está siendo agredido, que está en riesgo o lo que usted quiera, esas son las dos formas que se pueden actuar, para poder proteger una menor. (Oficial de policía, agosto, 2018)

Dentro de la Dinaf, la institución rectora del sistema, los niños, niñas o adolescentes ingresan por medio de denuncias de maltrato o a través de la remisión de un caso por parte de otra institución gubernamental o no gubernamental. «Entonces les informamos a ellos [la Dinaf], con copia de la denuncia, con copia de la investigación o indicándoles dónde se encuentran ubicados los niños para que aquellos puedan encontrar la manera de protegerlos, rescatarlos y dejarlos bajo su protección» (Fiscal, marzo, 2018).

Hasta este momento, cuando alguien llega con una denuncia, se atiende en el área de filtro; en el área de filtro se le escucha al usuario, que puede ser un niño, un adulto, un líder 
de comunidad, un centro hospitalario; o sea, de alguna manera que nos permita a nosotros abrir un expediente. En el área de filtro se abre expediente si es una competencia de la Dinaf. Si es una competencia de la Dinaf el caso, se remite al departamento legal que está contiguo al área de filtro; en la regional así funciona. (Directivo Dinaf, septiembre, 2018)

\section{Medidas implementadas}

En la figura 2 se puede apreciar el proceso que sigue la Dinaf antes de determinar qué medida de protección se asignará al niño, niña o adolescente. Una de las medidas identificadas es el traslado del joven con un familiar cercano en caso de que su seguridad no esté garantizada en su residencia.

\section{Figura 2}

\section{Algunas medidas de protección identificadas en la Dinaf}

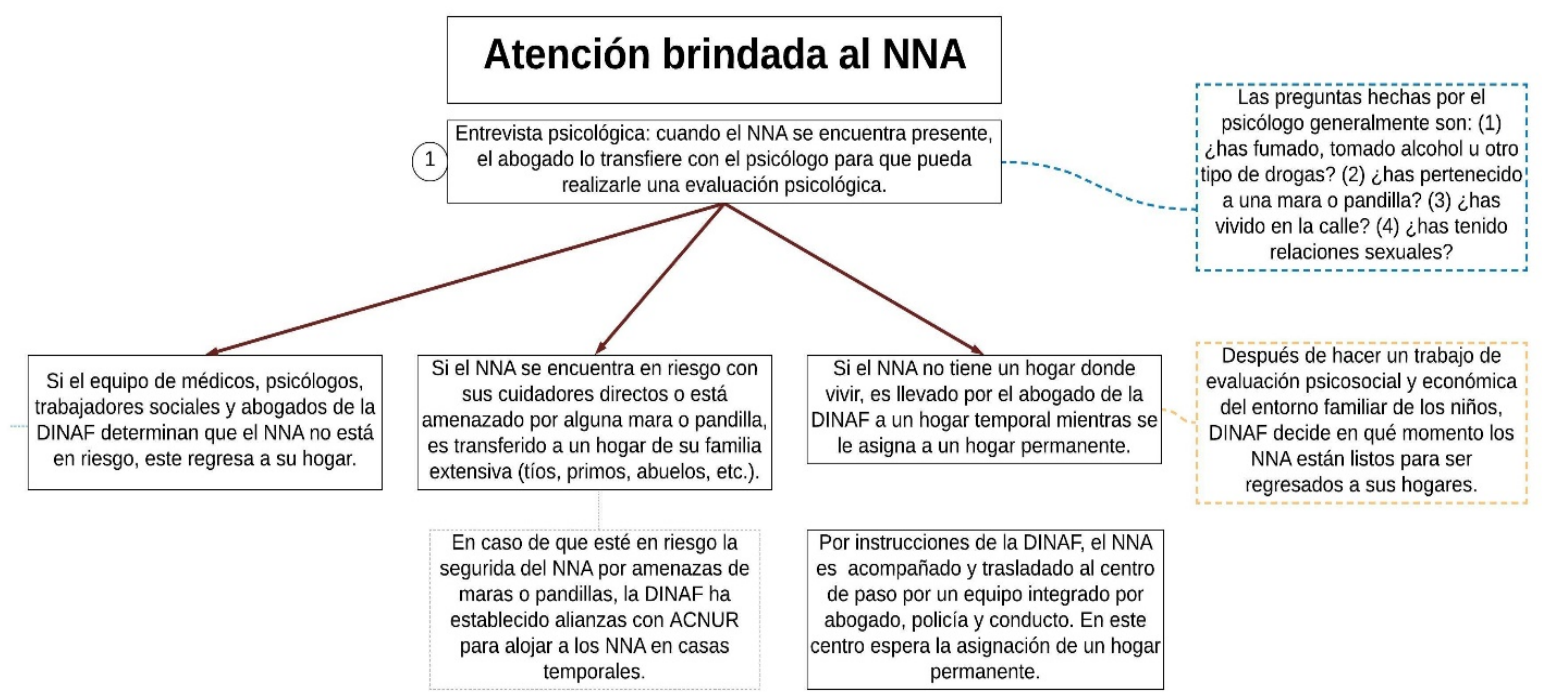

Lo que pasa es que con tal que no sea una familia que haya tenido conocimiento de esa agresión o que haya sido víctima la niña; por eso usamos lo que se llama «familia extendida», que es tu primo, tu tío, tu abuela. Entonces la ubicamos, tratamos de ubicarla con un familiar, no con una familia que no es biológica. (Abogado Dinaf, abril, 2018)

Así mismo, se encontró que la Dinaf puede asignar al joven a una familia temporal, que es un programa que paga a una familia por recibir en su hogar al niño o niña temporalmente; o bien se institucionaliza en una residencia transitoria llamada Centro de paso, la cual, generalmente, es un paso previo a una institución de residencia permanente; 
medidas que se aplican en los casos en que el niño, niña o adolescente no tenga un familiar con quien pueda vivir.

Yo lo recibo, lo presento a los niños, le pregunto que si tiene hambre, en la cocina [se] le da de comer y después yo me llevo al niño a que se bañe porque hay que revisar los golpes que trae (...). Ya le he entregado todo su kit, sus calcetines, su bóxer, todo esto después de la comida. Ya le asigné su espacio, su cama, su gavetero donde va a poner su ropa. (Trabajador, institución de residencia, julio, 2018)

Nuestro trabajo es trabajar el carácter de las personas. Trabajamos [con] adolescentes, niños, niñas, madres y adultos. Yo trabajo solo [con] adolescentes niñas. Hay siete hogares que se dividen en lo que le dije, adolescentes, niños, niño, adultos, madres. Se trabaja la adicción, la prevención y el carácter. (Psicóloga, institución de residencia, junio, 2018)

Otra medida de la Dinaf en caso de que, de manera definitiva, el niño, niña o adolescente no pueda regresar con su familia es iniciar un proceso de adopción, en el cual una familia nacional o extranjera, que cumpla los requisitos establecidos por la Dinaf, asume la responsabilidad de él o ella.

Se trabajan con el departamento de consolidación familiar; es un departamento ya específico solo para dar esa atención, de abandono y adopciones. Entonces ellos buscan la familia para adopciones y ven las mejores alternativas que pueda tener, ya sea nacional, con familia nacional o internacionales, verdad. (Abogado Dinaf, abril, 2018)

\section{Seguimiento de medidas}

El equipo de la Dinaf puede realizar visitas domiciliarias de evaluación multidisciplinaria a las familias o centros de residencia en los que se encuentre el niño o niña.

Posteriormente, para dar una primera visita supervisada con sus familiares o padres que no hayan vulnerado sus derechos, es después de 2 meses. (Abogada Dinaf, septiembre, 2018)

\section{Salida del sistema}

Principios como el criterio de oportunidad — como se verá más adelante — son medidas que pueden cerrar el proceso de manera temprana. «Ahora hay casos que se pueden resolver de inmediato en el cual las circunstancias lo permiten» (Directiva Dinaf, septiembre, 2018). 
Supóngase que es un tío que llega a la casa de su hermana y maltrata al niño. En esas condiciones generalmente termina esa denuncia con un criterio de oportunidad, porque es algo esporádico, es algo en el cual el agresor no es el encargado directo de brindarle protección al niño, sino que se toma como que fuera un particular. (Fiscal, marzo, 2018)

Por otra parte, la posibilidad de vivir con un familiar responsable o la culminación del proceso judicial que involucre al niño, niña o adolescente son acontecimientos que permiten culminar con la medida de protección a cargo del Estado y demás organizaciones involucradas. Así mismo, el cumplimiento del período de institucionalización o el cumplimiento de los 18 años de los niños, niñas o adolescentes son eventos que pueden ser motivo de culminación del proceso. «El año pasado salieron tres, antepasado salieron dos. Es difícil que termine una porque no hay apoyo de los padres» (Psicóloga, institución de residencia, junio 2018).

Es que, por ley, lo ponen de 18 para abajo; entonces una vez que están en 18 para abajo, se supone que ya debieron, lo ideal, lic, que cuando lleguen a esa etapa de los 18 años, ellos ya deben ser ciudadanos, estudiados, que puedan ser capaces de salir adelante, verdad. (Abogado, Dinaf, abril, 2018)

\section{Prácticas institucionales}

\section{Facilitación de la protección de derechos}

En el proceso de ingreso al sistema se identificó que existen múltiples formas de denuncia de casos de maltrato, ya sea por medio de llamada o de manera presencial. Se encontró que, en el caso de los centros educativos, existe una estrategia de intervención temprana por parte de profesores y autoridades escolares para identificar posibles maltratos y apoyar al niño o niña para iniciar un proceso que garantice su protección. También se observó que, entre las instituciones responsables de iniciar con los procesos de investigación ante una denuncia de maltrato (Dinaf, Ministerio Público, Policía Nacional) existe comunicación fluida, permitiendo acelerar los procesos necesarios para brindar una protección más efectiva. «Definitivamente tienen que pasar por la Dinaf porque es el ente rector de la niñez. Hacen una evaluación de la problemática del niño y lo refieren a nosotros» (Psicóloga, institución de residencia, junio 2018). De igual forma, se identificó que las instituciones mencionadas cuentan con un(a) psicólogo(a) para ofrecer este tipo de atención a los niños, niñas o adolescentes. «Se hacen evaluaciones forenses, psicológicas que se hacen por parte del departamento de medicina forense para determinar el delito y tomar ellos acciones» (Psicóloga, Dinaf, septiembre, 2018). Por parte de 
la Policía Nacional existe la práctica de apartar a los y las jóvenes de establecimientos en donde estén en situaciones de abuso o explotación laboral.

En relación con las medidas de protección aplicadas, se identificó que en el Ministerio Público se utiliza el criterio de oportunidad. Este criterio es aplicado solamente en casos que ocurren de manera única por parte de un familiar externo al niño o la niña pues, si el infractor es responsable directo, este es sometido a un proceso judicial; «pero cuando son las personas pendientes, que sí tienen la obligación de cuidar todos esos niños, en esos casos sí se presentan requerimientos contra ellos» (Fiscal, marzo, 2018). En relación a la Dinaf, se identificó que, cuando determina que el o la joven no puede vivir en su hogar de origen por cuestiones de seguridad, este es trasladado con el familiar más cercano con la capacidad de cuidarlo, es asignado a una familia temporal o, en última instancia, es trasladado a una institución de residencia. Este tipo de instituciones reciben un subsidio por parte de la Dinaf para cubrir parte de los costos del personal a su cargo y de las necesidades de los niños y niñas que atienden.

Existe un modelo de familia de protección temporal. Existen familias solidarias reciben un pago que... casi simbólico creo que son como 2500 lempiras ${ }^{2}$ por niño al mes y el resto pues ellos los tienen que cubrir y estos niños pueden ingresar a estos sistemas de protección antes de ingresar a una familia. (Abogada, Dinaf, marzo, 2018)

Una importante práctica dentro de los centros de residencia es que tratan de dar continuidad a la educación de los niños y niñas, componente que forma parte de sus rutinas cotidianas, aunque estas solo cubren las clases de español y matemáticas correspondientes al nivel educativo en el que se encontraban antes de ingresar en esta institución. «[De] 8:00 a 10:00 tienen la primera clase de reforzamiento que es español y matemáticas. Es donde más se refuerza porque es donde más problemas hay. La profe les va revisando, tienen ejercicios de ortografía» (Trabajador, institución de residencia, julio, 2018). Finalmente, en cuanto al proceso de monitoreo y seguimiento de las medidas de protección, se encontró que la Dinaf envía a su personal con las familias e instituciones que tienen niños y niñas bajo su responsabilidad.

\section{Obstaculización de la protección de derechos}

En torno al trabajo realizado, las y los participantes señalaron condiciones de la infraestructura del sistema de protección que dificultan el tipo y la calidad de los servicios

${ }_{2}^{2}$ Aproximadamente USD 100.0o. 
que brindan. Una de ellas, y quizás la más importante, es el limitado presupuesto con el que cuentan las instituciones, pues se carece de los recursos necesarios para realizar su trabajo cotidiano. Ejemplo de ello es que en algunas ocasiones deben buscar fondos externos para poder financiar sus actividades. «Nos toca pues abrirnos puertas y pues, si en ese momento la institución no tiene recursos y tenemos que hacer una actividad, pues toca hacer nosotros, hacer esa coordinación» (Directiva, Dinaf, septiembre 2018). Igualmente, estas instituciones no se encuentran en todo el territorio del país, reflejando desigualdades en el acceso, principalmente para la población residente en las zonas rurales. Una de las personas entrevistadas confió la responsabilidad en estas zonas a ONG.

Se está revisando, y la meta, verdad, de la dirección ejecutiva, es esa: tener centros de paso a nivel nacional. Pero a veces no es necesario porque realmente se cuenta con alrededor de 30-40 ONG que nos dan ese tipo de ayuda a lo largo del territorio nacional. (Abogado, Dinaf, abril 2018)

Dentro de la labor de investigación del Ministerio Público se encontró que los fiscales tienen bajo su responsabilidad una elevada carga de trabajo, impidiendo que todos los casos puedan ser investigados. «No hay logística de vehículos, no hay suficientes agentes, e igual nos pasa a nosotros, verdad; nosotros aquí, el que menos cantidad de denuncias tienen en su poder para investigar son 1500» (Fiscal, marzo, 2018). Por último, se recibió información de que los procesos de selección del personal de estas instituciones, principalmente las que trabajan directamente para el Gobierno - como es el caso de la Dinaf- se encuentran altamente politizados, ya que priman como criterio de selección la pertenencia al partido de gobierno y no la experiencia o la formación académica. «Porque eso influye también, la política, porque esta institución no debería de ser política; sin embargo, no entra nadie que no haya pasado el filtro; ${ }^{3}$ si no pasó el filtro, no puede entrar» (Profesor universitario, mayo, 2018).

En cuanto a la primera etapa - es decir, el ingreso-, una de las prácticas que obstaculizan la protección de los derechos es la utilización de la policía por parte de familiares a fin de llevar a niños, niñas o adolescentes ante la Dinaf que, por sus zonas de residencia, tienen contacto o simpatizan con maras y pandillas, aunque no hayan cometido ningún delito.

${ }^{3}$ «Filtro» en este caso significa pertenecer al partido de gobierno. 
Eran como las 10 de la noche cuando vinieron los policías y hasta mi mami vino enojada y dijo que, si yo me escapaba, me iban a poner en un centro donde tenía que trabajar y que no iba a comer. (Adolescente, institución de residencia, junio, 2018)

Por otra parte, niños y niñas tienen que asistir a múltiples instituciones, y en cada una de ellas son sometidos por diferentes instancias a un proceso de exhaustivas entrevistas hasta culminar con un(a) psicólogo(a). Esta forma de intervención tan segmentada produce una revictimización, dado que en cada etapa, al ser entrevistados, tienen que revivir la experiencia negativa que les condujo al sistema.

Primero le cuentan a su mamá o su papá, después le cuentan a la gente que hace la entrevista original, después vienen y le cuentan lo mismo al forense, le cuentan lo mismo al psicólogo, al psiquiatra y todavía lo llaman a juicio oral a declarar ante el tribunal de sentencia; es una revictimización increíble. (Fiscal, marzo, 2018)

Con relación a la etapa en la cual se implementan las medidas de protección, se identificó que en una de las instituciones de residencia asignan a los niños, niñas o adolescentes responsabilidades de limpieza de las instalaciones. En uno de estos centros, se encontró lo que parece ser una práctica sistemática de discriminación contra niños, niñas o adolescentes pertenecientes a la comunidad LGTBIQ, a quienes no se les brinda acogida por considerar que su presencia pone en riesgo el bienestar del resto de residentes.

Se le llama al director o subdirector y ellos ya se encargan de dar los motivos por los cuales el niño (LGTBIQ) no puede estar aquí. No podemos vulnerar el derecho de siete niños por salvar el derecho de un niño y aparte poner en peligro a los demás. (Trabajador, institución de residencia, julio, 2018)

Por otra parte, dentro de las instalaciones de una de las residencias se encontró la presencia de militares, quienes realizan trabajo de vigilancia y son responsables por la seguridad. «Hay seguridad suficiente, aparte de que tenemos los dos militares» (Trabajador, institución de residencia, julio, 2018). Finalmente, se halló que los adolescentes salen de estas instituciones automáticamente al cumplir 18 años, lo cual, aunque se fundamente en la ley, no garantiza que el joven esté en disposición económica, física o emocional para poder salir de la misma. 


\section{Discusión}

En el presente documento se ha tratado de exponer, a través de sus diferentes etapas e instituciones involucradas, las prácticas institucionales que facilitan y obstaculizan la protección de los derechos de la niñez en el sistema de protección hondureño. Los resultados expuestos señalan que en las etapas de ingreso al sistema y la implementación de las medidas de protección es donde hay un mayor número de prácticas que facilitan la protección de las y los niños. Entre ellas se destaca la colaboración existente entre las diversas instituciones que constituyen el sistema, la disposición de servicios psicológicos en cada una de las instancias a las que deben acudir los usuarios, así como también la variedad de estrategias que pueden adoptar los proveedores de servicios para proteger los derechos de los niños y niñas.

Por otra parte, existen condiciones de infraestructura que dificultan el alcance que puede tener el sistema de protección en el logro de sus objetivos, siendo la limitación de recursos que las diferentes instancias tienen a su disposición una de las más importantes. Entre las prácticas que obstaculizan la protección de los derechos de los niños, niñas y adolescentes destaca la presencia de fuerzas de seguridad en diferentes etapas del proceso, al igual que la identificación de prácticas discriminatorias contra la comunidad LGTBIQ entre el personal de una de las instituciones visitadas. Lo anterior muestra que las prácticas institucionales no se guían exclusivamente por normas legales, sino también de creencias y prejuicios que construyen a los niños, niñas y adolescentes de esta comunidad como una amenaza.

Una de las limitaciones del presente trabajo es que los datos están referidos a las instancias visitadas; por esta razón se considera necesario complementarlo con posteriores estudios que permitan ampliar el número de instituciones analizadas. Por otro lado, dada la escasez de estudios académicos sobre el sistema de protección hondureño, se trató de abarcar una significativa extensión de procesos con el propósito de exponer una visión integral, en detrimento de la profundización en cada una de las experiencias o instituciones mencionadas. Así también, resulta necesario hacer hincapié en que los resultados expuestos son preliminares, pues la investigación de la que forman parte todavía se encuentra en proceso de realización (en sus etapas posteriores).

Dicho lo anterior, se concuerda con estudios previos en que todavía persisten importantes grietas entre lo establecido en la Convención sobre los Derechos del Niño y la 
protección efectiva que reciben los niños, niñas y adolescentes (Martínez-Franzoni, 2014; Olivares \& Reyes, 2016, Parada, 2011; Parada et al., 2007; Sola-Morales \& Campos-Garrido, 2019; Villalta \& Llobet, 2015; Visión Mundial, 2014). Esto se ve reflejado en la escaza disposición de recursos que adolecen las instituciones del sistema de protección, aun cuando en la Convención los Estados se comprometen a utilizar todos los recursos a su alcance para garantizar la protección de sus derechos (Convención sobre los Derechos del Niño, art. 4). Así mismo, resulta paradójico constatar que dentro de las instancias responsables de la protección de los niños, niñas y adolescentes existan actitudes discriminatorias por motivo de orientación sexual o identidad de género, cuando el principio de no discriminación es un pilar fundamental de la Convención (Organización de las Naciones Unidas, 1989, art. 2).

Por otra parte, se concuerda con otros autores (Martínez-Franzoni, 2014; Oficina Internacional de los Derechos del Niño, s. f.; Olivares \& Reyes, 2016; Parada, 2011) en que muchas prácticas se fundamentan en concepciones tradicionales de la infancia; es decir, los niños y niñas son vistos como objetos de intervención, como «menores» o como problema, en vez de ser vistos como sujetos a quienes se les deben garantizar sus derechos o como agentes capaces de participar en los problemas que les afectan. Al respecto, se concuerda con Olivares y Reyes (2016), para quienes los cambios de perspectiva en el abordaje institucional de la niñez, «solo repercute de manera discursiva, manteniendo aún resabios de la perspectiva tutelar» (p. 442).

Lo anterior se identifica en el tratamiento que reciben niños y niñas por parte de fuerzas de seguridad en diferentes etapas del proceso de protección, donde la escolta y vigilancia permanente de policías y militares se asemeja a los procedimientos propios del sistema penitenciario. En este sentido, se coincide con Rosier (2009), quien afirma «bad children themselves threaten the safety of good children and adults alike, and here again tremendous amounts of time, energy and money are devoted to their publication, prosecution and incarceration» ${ }^{4}$ (p. 267), puesto que son los niños, niñas o adolescentes que se encuentran en mayor condición de vulnerabilidad por su cercanía con las maras y pandillas los tratados más como responsables que como víctimas de su situación; es decir, como un problema que amerita la intervención del Estado y no como sujetos a quienes no se les ha garantizado su derecho a la vida. Corsaro (2018, p. 277) denomina «culpabilizar

\footnotetext{
${ }^{4}$ «Los propios niños malos amenazan la seguridad de niños y adultos buenos, y aquí nuevamente se dedican enormes cantidades de tiempo, energía y dinero a su reporte, procesamiento y encarcelamiento.»
} 
a la víctima» a esta tendencia de responsabilizar a los niños y niñas por las condiciones estructurales que los han llevado a la vulnerabilidad en la que se encuentran.

Ahora bien, ante un escenario de precariedad económica y social crítica, ¿qué papel puede jugar el sistema de protección de la niñez? El trabajo del sistema de protección parece desbordado pues, aunque existen niños y niñas que se benefician de los servicios prestados por las instituciones del sistema, existe un número mayor que queda desatendido; por tanto, el sistema no es capaz de contener las violaciones a los derechos provocadas por el contexto social, por ejemplo, en materia de garantía a la supervivencia y el desarrollo. Aún más, las propias instituciones del sistema de protección no cuentan con los recursos suficientes para atender a la población que los visita y que representa solamente un porcentaje minúsculo de niños, niñas y adolescentes del país. En consecuencia, ante este escenario es factible cuestionar si la suscripción de los Estados a la Convención, así como la creación formal de un sistema de protección, no funge más como un recurso ideológico utilizado tanto para encubrir la potencial crítica a las condiciones estructurales que generan la vulnerabilidad de los niños y niñas, como para abordar el problema de la protección desde un enfoque de casos individuales, desconectados de las condiciones sociales de las que forman parte (Rensmann, 2017).

Con esto no se duda de la importancia que tiene la Convención como paradigma jurídico para orientar las políticas estatales dirigidas a esta población; al contrario, se considera que esta es un mecanismo significativo para presionar a los Estados a que mejoren las condiciones de vida de los niños y niñas (Rensmann, 2017). Sin embargo, es necesario tener en cuenta que la adscripción retórica a los principios de la Convención, tal como ha quedado demostrado, no es suficiente para garantizar su bienestar (Di Loro, 2010). De esta manera, haciendo eco de las ideas de Villata y Llobet (2015), cabe preguntarse si los obstáculos identificados tanto en el contexto nacional como en las instituciones que conforman el sistema de protección de la niñez en Honduras, más que un telón de fondo que condiciona negativamente el funcionamiento de las diferentes instancias, son la forma de funcionar del sistema mismo.

\section{El Código de la Niñez y la Adolescencia}

Es necesario recordar que el Código de la Niñez y la Adolescencia es el marco legislativo que ha regido todos los esfuerzos del país en materia de niñez; primero entre 1996 y 2013, año en que el gobierno de turno llevó a cabo una serie de reformas a las leyes del país en materia de niñez; y un segundo término desde el 2013 en adelante. Para algunos 
autores (Dávila \& Naya, 2012) el Código anterior cumplía con un alto grado de congruencia en relación con la Convención. Situación que resulta paradójica, ya que es solo con las nuevas reformas realizadas en 2013 que se incorpora, por ejemplo, la expresión «interés superior del niño» (en el artículo 5), elemento a tomarse en cuenta en todas las disposiciones administrativas, civiles y penales relacionadas con la niñez y adolescencia. Así mismo, es solo a partir de las nuevas reformas que se introduce explícitamente la concepción de la niñez como «sujetos de derechos». Y en consonancia con tales incorporaciones, a la palabra «niño» en el Código, se le agrega la palabra «y niña» para referirse a todos y todas los que entran dentro de la categoría general de niñez.

Aunque se celebran las incorporaciones, se identifican algunos problemas en relación con el Código actual. Por ejemplo, debido a que las mencionadas reformas se realizaron un año antes de la creación de la actual instancia rectora del sistema de protección, el Ihnfa todavía figura en la redacción de sus artículos. Situación que puede representar problemas no menores en el ámbito jurídico, puesto que las facultades entre la antigua y nueva instancia rectora varían considerablemente. De igual forma, una de las principales consecuencias de tales reformas fue el distanciamiento del protagonismo del Estado hondureño en la protección de los derechos de la niñez en el país. A partir de ellas se consolidan principios como la descentralización y tercerización que han tenido consecuencias en tres áreas importantes: 1) degradación del rango de la instancia rectora del sistema de protección, al pasar de ser una institución autónoma (Ihnfa), a una dirección subordinada a la Secretaría de Desarrollo e Inclusión Social (Dinaf); 2) transferencia de la responsabilidad de la protección de la niñez las municipalidades y ONG que trabajan en el país; y 3) reducción del presupuesto estatal invertido en niñez y adolescencia.

\section{Consideraciones finales}

Aunque es posible que quede trabajo por hacer para comprender en profundidad las prácticas institucionales que facilitan u obstaculizan la protección de la niñez en el sistema de protección hondureño, se considera que este artículo representa una aproximación integral que permite comprender su funcionamiento en líneas generales. Ejercicio que puede contribuir al campo académico en un doble sentido: por un lado, del trabajo realizado por Voltarelli et al. (2018) se desprende que los estudios de la niñez realizados desde Centroamérica no figuran en el subcampo de la sociología de la infancia en el mundo hispano-hablante; por lo que esta investigación vendría a aportar mínimamente en la consolidación de este campo en el Istmo. Por otro lado, al hacer uso de la etnografía 
institucional como método para acceder a las realidades de las diferentes instancias del sistema de protección de la niñez en Honduras, el trabajo contribuye con nuevas propuestas metodológicas para abordar los estudios de niñez, la cual se encuentran en plena consonancia con los principios del enfoque constructivista de la sociología de la infancia (Gaitán-Muñoz, 2006).

En cuanto al contexto estatal, en donde usualmente se cree que la mera existencia de leyes y reglamentos garantiza el funcionamiento de las instituciones, esta investigación se presenta relevante en tanto ejercicio reflexivo acerca de las propias prácticas, ya que, al distanciarse del quehacer cotidiano que tiende a absorber a los trabajadores y usuarios del sistema, este tipo de estudios permiten identificar procedimientos y acciones que podrían estar perjudicando a aquellos que oficialmente se afirma proteger.

\section{Agradecimientos}

Esta investigación se realizó gracias al financiamiento del Consejo de Investigación en Ciencias Sociales y Humanidades de Canadá (SSHRC, siglas en inglés). Se agradece al equipo de investigación de la Escuela de Trabajo Social de la Universidad de Ryerson por la revisión y observaciones realizadas al borrador del presente documento.

\section{Referencias}

Alzate-Piedrahíta, M. V. (2003). La infancia: concepciones y perspectivas. Repositorio UTP. https://bit.ly/2TQJPIj

Banco Mundial. (2019). Informe anual. https://www.bancomundial.org

Corsaro, W. A. (2018). The sociology of childhood. Sage.

Dávila, P., \& Naya, L. M. (2012). La protección de la infancia en situaciones de alto riesgo en América Latina a través de los códigos de la niñez. Pedagogía Social. Revista Interuniversitaria, (19), 99-112. https://doi.org/10.7179/psri_2012.19.08

Devault, M. (2006). Introduction: What is institutional ethnography? Social Problems, 53(3), 294-298. https://doi.org/10.1525/sp.2006.53.3.294

Di Loro, J. (2010). Infancia e institucionalización: abordaje de problemáticas sociales actuales. Pesquisas e Prácticas Psicossociais, 4(2), 143-150. 
Dirección de Niñez, Adolescencia y Familia. (2015). Plan estratégico de la Dinaf. https:// bit.ly/2Zy5w3b

Gaitán-Muñoz, L. (2006). La nueva sociología de la infancia: aportaciones de una mirada distinta. Política y Sociedad, 43(1), 9-26.

Instituto Universitario en Democracia, Paz y Seguridad. (2018). Mortalidad y otros. Universidad Nacional Autónoma de Honduras.

Martínez-Franzoni, J. (2014). Protección social para la infancia en El Salvador, Guatemala y Honduras. Cepal, Unicef. https://bit.ly/2ASzowY

Mayall, B. (2001). The sociology of childhood in relation to children's rights. The International Journal of Children's Rights, (8), 243-259.

Mcreery-Bunker, K., Groza, V., \& Lauer, D. P. (2009). International adoption and child protection in Guatemala. International Social Work, 52(5), 649-66o. https://doi.org/ $10.1177 / 0020872809337676$

Mieles, M. D., \& Acosta, A. (2012). Calidad de vida y derechos de la infancia: un desafío presente. Revista Latinoamericana de Ciencias Sociales, Niñez y Juventud, 10(1), 205-207.

Observatorio Demográfico Universitario. (2018). Geoportal. http://geoportal.odu.unah.edu.hn/

Oficina Internacional de los Derechos del Niño. (s. f.) Mapeo del sistema de protección de la niñez y adolescencia en Honduras. https://bit.ly/2LVwtGb

Olivares, B., \& Reyes, E. (2016). Evaluación de un programa social en infancia desde los principios de la Psicología Comunitaria. Revista Latinoamericana de Ciencias Sociales, Niñez y Juventud, 14(1), 431-444. https://doi.org/10.1160o/1692715x.1417090915

Organización de las Naciones Unidas. (1989). Convención sobre los Derechos del Niño. Autor.

Parada, H. (2011). Prácticas institucionales en el Sistema de Protección de Niños, Niñas y Adolescentes: etnografía institucional. Unicef.

Parada, H., Moffatt, K., \& Duval, M. (2007). Emerging practices of social work in the Dominican Republic. International Social Work, 5o(6), 755-769. https://doi.org/ 10.1177/0020872807081902

Prout, A., \& James, A. (1997). A new paradigm for the sociology of childhood? Provenance, promise and problems. En A. James, \& A. Prout (Eds.), Constructing and reconstructing childhood (pp. 7-32). Falmer Press.

Rensmann, L. (2017). Critical theory of human rights. En M. J. Thompson (Ed.), The Palgrave handbook of critical theory (pp. 631-653). Palgrave McMillan. https://doi.org/ 10.1057/978-1-137-55801-5

República de Honduras. (1996). Decreto №. 73. Diario Oficial La Gaceta. https:// www.oas.org/dil/esp/Codigo_Ninez_Adolescencia_Honduras.pdf 
República de Honduras. (1998). Decreto №. 199. Diario Oficial La Gaceta. http://

www.sipi.siteal.iipe.unesco.org/sites/default/files/sipi_normativa/

honduras_decreto_nro_199_1997.pdf

República de Honduras. (2014). Decreto Ejecutivo PCM 26. Diario Oficial La Gaceta.

http://extwprlegs1.fao.org/docs/pdf/hon138149.pdf

Rosier, K. B. (2009). Children as problems, problems of children. En J. Qvortrup, W. A.

Corsaro, \& M.-S. Honig (Eds.), Tha Palgrave handbook of children studies (pp. 256-272).

Palgrave McMillan.

Santos, D. M. (2017). Subsistema de protección especial de derechos de niñas, niños y adolescentes: marco conceptual y de funcionamiento, Honduras. Dirección de Niñez, Adolescencia y Familia.

Saunders, F., Zepeda, S., Interiano, L., \& Andino, S. (2014). Pobreza infantil en Honduras: análisis de progresividad y redistribución de las transferencias dirigidas a la niñez. Revista Economía y Administración (E\&A), 5(2), 126-149. https://doi.org/10.5377/ eya.v5i2.2187

Sola-Morales, S., \& Campos-Garrido, N. A. (2019). Discurso estatal chileno en la protección de los derechos de niños(as) y jóvenes. Revista Latinoamericana de Ciencias Sociales; Niñez y Juventud, 17(1), 105-204. https://dx.doi.org/10.1160o/1692715x.17106

Strauss, A., \& Corbin, J. (2002). Bases de la investigación cualitativa: técnicas y procedimientos para desarrollar la teoría fundamentada. Editorial Universitaria de Antioquia.

Unicef. (2016). Garantizar los derechos de la niñez y adolescencia vulnerable en Honduras. Avances y desafíos en la construcción de un sistema integrado de protección social. Unicef.

Urzin, M., Oltedal, S., \& Muñoz, C. (2017). Recognizing the «big things» and the «little things» in child protection cases. Child \& Family Social Work, 22(2), 932-941. https:// doi.org/10.1111/cfs.12313

Villalta, K., \& Llobet, V. (2015). Resignificando la protección: los sistemas de protección de derechos de niños y niñas en Argentina. Revista Latinoamericana de Ciencias Sociales, Niñez y Juventud, 13(1), 167-180.

Visión Mundial. (2014). Los sistemas de protección de la niñez en América Latina y el Caribe: un análisis a nivel nacional y comunitario en 10 países. Visión Mundial. https://bit.ly/3eelxPT Voltarelli, M. A., Gaitán-Muñoz, L., \& Leyra-Fatou, B. (2018). La sociología de la infancia y Bourdieu: diálogos sobre el campo en los países hispano-hablantes. Política y Sociedad, 55(1), 283-309. https://doi.org/10.5209/POSO.56119 